\title{
PROPUESTA DE GESTIÓN TERRITORIAL DEL COMPLEJO ECOLÓGICO ASOCIADO CON LA LAGUNA CARIMAGUA, A PARTIR DE UNA EVALUACIÓN DE SERVICIOS ECOSISTÉMICOS: MUNICIPIO DE PUERTO GAITÁN-META
}

\section{Proposal for Territorial Management Associated Complex Ecological Lagoon Carimagua, From an Assessment of Ecosystem Services: Municipality of Port Gaitan -Meta}

\author{
DIANA C. TORRES1, BRIGITTE BAPTISTE², CLARA CARO 3 . \\ ${ }^{1}$ Bióloga, MSc. Gestión Ambiental Sostenible, Universidad de los Llanos, Villavicencio, Meta, Colombia. \\ ${ }^{2}$ Bióloga, MSc. en Conservación y Desarrollo Tropical, Instituto Alexander Von Humboldt, Bogotá, Colombia. \\ ${ }^{3}$ Bióloga, MSc en Ecología, Universidad de los Llanos, Villavicencio, Meta, Colombia.
}

E-mail: dctorresm@gmail.com brigittebaptiste@humboldt.org.co

clarainescaro@unillanos.edu.co

Aceptado: 5 de Mayo de 2014

\section{Resumen}

El complejo ecológico asociado a la laguna Carimagua localizada en el departamento del Meta, Colombia es considerada una estrella fluvial de importancia binacional asociada al a cuenca del Orinoco. Está zona se caracteriza por la prestación de servicios ecosistémicos de gran importancia para garantizar la sostenibilidad de las comunidades, los cuales fueron evaluados desde los componentes social, ecológico y económico con el fin de generar un mecanismos de gestión territorial fundamentado en la relación socioecosistémica buscando la Conservación de la zona mediante la propuesta de creación de un área protegida en el marco del decreto 2372 de 2010 bajo la categoría de Distrito de manejo integrado.

Palabras claves: Servicios ecosistémicos, Laguna Carimagua, Fragstats, Valoración contingente y Percepciones sociales.

\section{Abstract}

The ecological complex associated Carimagua lagoon located in the department of Meta, Colombia is considered an important binational waterway star associated to the Orinoco basin. It's area is characterized by the provision of ecosystem services important to ensure the sustainability of communities, which were evaluated from the social, ecological and economic components in order to generate mechanisms of territorial management based on the socioecosistemic relationship seeking conservation area through the proposed establishment of a protected area in under the decree 2372 of 2010 under the category of integrated management District area.

Keywords: Ecosystem Services, Laguna Carimagua, Fragstats, Contingent valuation and social perceptions. 


\section{INTRODUCCIÓN}

La visión integral de la biodiversidad implica el reconocimiento de genes, especies, poblaciones y comunidades con el fin de evaluar sus interacciones, vistas como una unidad funcional: Ecosistemas (Balvanera 2009).

Los ecosistemas son considerados en la actualidad como fuente de distribución de recursos y propósito de gestión desde la planificación (Balvanera 2009), haciéndose evidente la necesidad de diagnosticarlos y evaluarlos con fines de conservación (*). Para ello, es necesario la comprensión de su composición, estructura y función, con el fin de integrar valores sociales, culturales y económicos, los cuales toman un papel protagónico e indispensable para garantizar la supervivencia del planeta (Laykea et al. 2012).

Así, se reconoce que los "sistemas naturales brindan el sostenimiento de la vida, como de la biodiversidad que estos albergan" (Prager et al. 2011). La transformación de bosques, selvas y pastizales naturales en sistemas de producción intensiva de bienes ha modificado la dinámica natural del medio y su capacidad para brindar otros beneficios tales como el intercambio de oxígeno y la contribución de los bosques a la regulación del clima o el control de la erosión. La búsqueda de satisfacción de las necesidades ha minimizado la capacidad que tienen los sistemas naturales para mejorar la calidad de vidas con los servicios prestados (Gámez et al. 2008).

Sin embargo, los vínculos entre el bienestar humano y los ecosistemas no se conocen a fondo para la gran variedad de contextos socio-económicos, y múltiples escalas desde lo global hasta lo local; el no evaluar adecuada e integralmente el territorio lleva a la toma de decisiones de ordenamiento inadecuadas, sin escenarios de partida reales afectando escalas locales, regionales y nacionales (Vila et al. 2006).

Es allí, cuando los avances temáticos muestran qué factores biofísicos y económicos deben integrarse a las miradas socioculturales, con el fin de construir líneas de conocimiento para el estudio de los servicios ecosistémicos y sus aplicaciones en la evaluación de ecosistemas neotropicales y la toma de decisiones en gestión ambiental (De Groot et al. 2002).

La Laguna Carimagua, los cuerpos de agua y la sabana plana son considerados ecosistemas de gran relevancia para la red hídrica de la cuenca del Orinoco por los procesos hidrológicos y ecológicos que se desarrollan y la diversidad biológica que sustenta. Debido a su gran riqueza hídrica actúan como una gran estrella fluvial, por lo que regula la dinámica hídrica de la zona, evitando la pérdida de Biodiversidad y garantiza la prestación de servicios ecosistémicos a comunidades localizadas aguas abajo y ecosistemas asociados a la gran cuenca.

En el presente trabajo se evalúan los servicios ecosistémicos del complejo ecológico asociado a la laguna Carimagua, desde una visión sistémica orientada a estructurar alternativas para su preservación y toma de decisiones enfocadas al ordenamiento ambiental territorial en la vereda Carimagua, municipio de Puerto Gaitán, Meta.

\section{ÁREA DE ESTUDIO}

El complejo ecosistémico conformado por la laguna de Carimagua $(480 \mathrm{Ha})$ y las áreas circundantes, se encuentra localizado en la hacienda Carimagua, en el extremo NorOriental del departamento del Meta, bajo jurisdicción del municipio de Gaitán a $105 \mathrm{Km}$ del casco urbano del municipio, limitando con el Vichada, tiene una extensión de aproximadamente 22000 Has, entre los 150 y 175 m de elevación (Figura 1).

\section{MATERIALES Y MÉTODOS}

La investigación se desarrolló en tres fases que permitieran identificar y evaluación de servicios ecosistémicos y generar una propuesta de gestión territorial así:

Fase I: Identificación de servicios ecosistémicos. Se identificaron los actores locales (pobladores: total de habitantes presentes en el área de estudio), clasificándolos por rangos de edad (18 - 29, 30 - 41, 42 - 53 y mayor a 53 años), a los cuales se les aplicó una encuesta enfocada en los servicios prestados por el ecosistema y se estableció su priorización con las escalas Alta, Media y conforme a la cantidad de veces que fue reconocido el servicio ecosistémico.

Fase II: Evaluación de los servicios ecosistémicos. Se evaluaron los tres servicios ecosistémicos priorizados, desde los componentes ecológico, económico y social así:

Ecológico: Se generaron polígonos georeferenciados en campo a (escala 1:25000) y digitalizados a partir de un juego de imágenes Landsat. Los límites de los parches fueron trazados en la fotointerpretación considerando la adyacencia con otros tipos de parches y las divisiones funcionales (carreteras, vías e infraestructura) que pueden restringir los flujos y movimientos entre parches (McGarigal et al. 2012).

El mosaico de parches creado por la fotointerpretación fue 
digitalizado en el SIG Arc View 3.2., georeferenciado y corregido a partir de coordenadas geográficas de puntos de control tomadas con un equipo GPS. Luego se cuantificó la heterogeneidad espacial del paisaje mediante el software Fragstats.
Económico: Se realizó una valoración económica parcial del servicio ecosistémico de existencia, mediante el método de valoración contingente, para lo cual, se realizaron encuestas en las cuales varío el valor de la disposición a pagar con respecto al ingreso económico del encuestado.

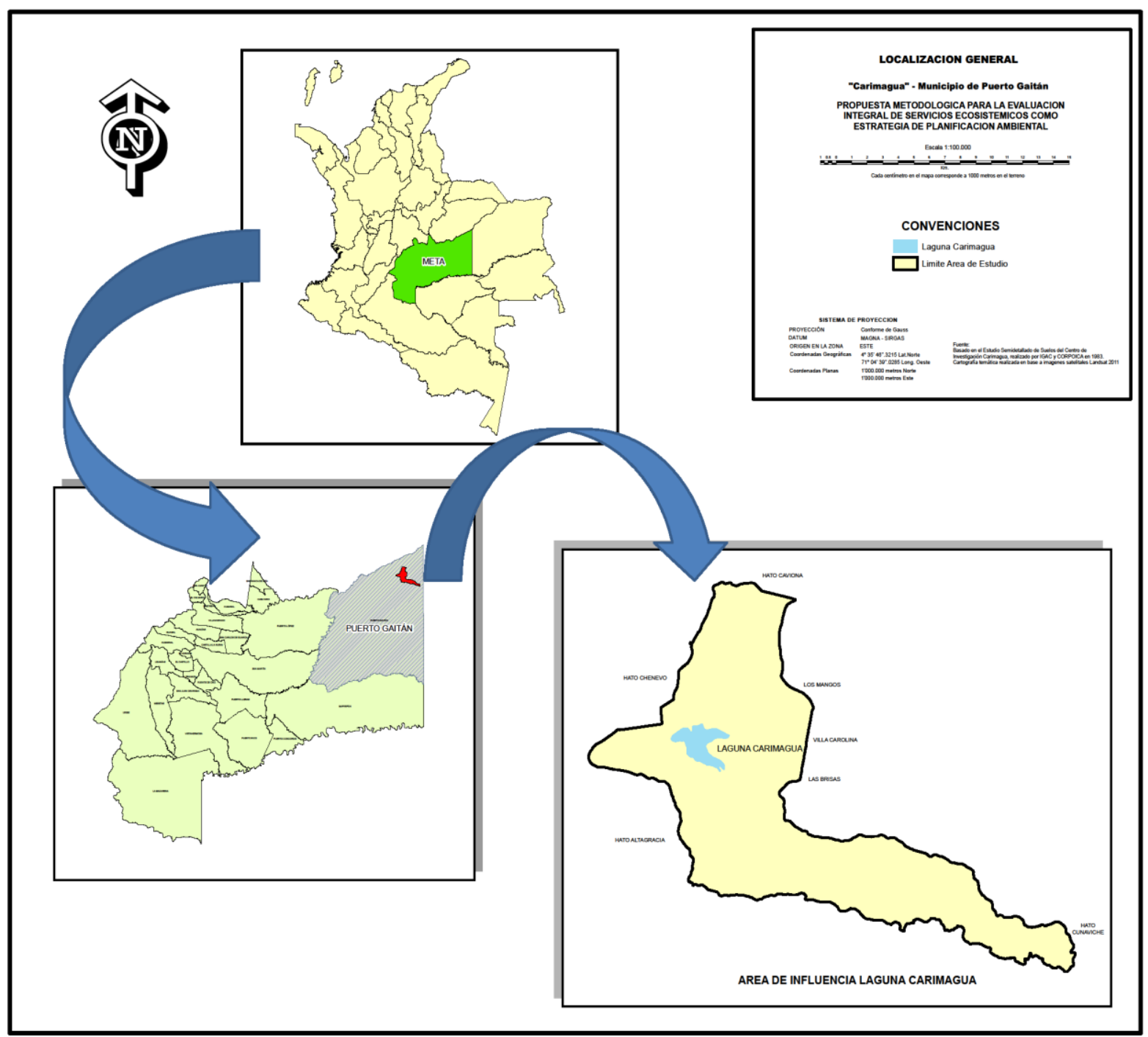

Figura 1. Localización del área del objeto de estudio, vereda Carimagua, Puerto Gaitán, Meta. Fuente: Elaboración propia.

Inicialmente, se elaboró y aplicó una encuesta piloto, la cual sirvió para determinar el valor que estarían dispuestos a pagar los habitantes de acuerdo a sus ingresos y con base en los resultados obtenidos se elaboraron las encuestas definitivas en las cuales varía el valor de la disposición a pagar en cada uno de los salarios. Debido al número limitado de pobladores de los predios, la población encuestada corresponde a la totalidad de habitantes asentados en la zona de influencia del complejo ecológico asociado a la laguna Carimagua.
La disponibilidad a pagar (DAP) se construyó a partir del análisis de los ingresos y egresos mensuales familiares, su recaudo se realizara mensualmente mediante la incorporación en un recibo de servicio público y su estimación se realizó mediante la especificación de un modelo de elección discreta Logit. Se codificó la variable dependiente como 1 si la respuesta a la disponibilidad a pagar es negativa y 0 si la respuesta es positiva. Posteriormente se corrieron los modelos de regresión logística lineal; logística logarítmica en las variables ingreso y monto de pago, mediante el programa de EconometricViews (E.Views 3.0). 
Social: Se evaluó el grado de uso y la percepción de vulnerabilidad de los servicios ecosistémicos por parte de los actores locales (todos los pobladores asentados en la zona de estudio) mediante una encuesta semi - estructurada donde se definieron tendencias en el uso de los recursos y estado en el territorio.

Fase III: Gestión y Planificación del territorio. Se aplicó la metodología de juego de roles "Manantiales de la sierra" (CEECO 2012) con el fin de realizar un análisis de la situación de los recursos naturales con respecto al uso del territorio.

En cuanto al uso del paisaje, se incluyeron las coberturas naturales de bosque de galería, sabanas y cuerpos de agua, usos de los paisajes para actividades productivas (ganadería, agricultura y extracción), determinando si los participantes valoran los servicios ecosistémicos que provee el complejo ecosistémico y se identificaron las preferencias de uso del paisaje, incluyendo actividades productivas mediante la definición de rol como dueños de finca con ganadería, agricultura y minería, representante del mercado (Cárdenas et al. 2013).

A partir de los resultados obtenidos previamente se revisaron la categoría de manejo del área conforme al decreto 2372 de 2010 aplicando un criterio de pertinencia al contexto territorial y necesidades locales para garantizar la prestación de los servicios ecosistémicos y la sostenibilidad del área, todo ello en función del bienestar humano.

\section{RESULTADOS Y DISCUSIÓN}

\section{Identificación de servicios ecosistémicos}

Se identificaron 105 habitantes contabilizados en los seis predios objeto de estudio, distribuidos en los siguientes rangos: el 30,48\% (32) menores de 18 años, el 16,19 \% (32) entre los 18 y los 29 , el 24,76\% (26) entre los 30 y 41, el 15,24\% (16) entre los 42 y 53 y 13,33\% (14) mayores a 50 años.

Los habitantes identificaron los servicios ecosistémicos traducidos a beneficios que recibe del área fundamentada en tres grandes grupos: los prestados por el Agua, la Sabana y los Bosques, obteniendo como servicios priorizados la calidad y cantidad del recurso hídrico, recreación, conocimiento científico y valor de existencia (Tabla 1).

En el área la identificación de los servicios ecosistémicos permitió evidenciar por parte de la comunidad el estratégico papel de la zona para la prestación de dos tipos de servicios ecosistémicos: culturales y de abastecimiento, los cuales fueron asociados a dos tipos de ecosistemas: acuáticos (Laguna, humedal y cuerpos de agua) y terrestres (Sabanas y Bosques).

Tabla 1. Servicios ecosistémicos identificados y priorizados para el complejo ecológico asociado con la laguna Carimagua. ${ }^{*}$ Numero de veces que fue nombrado el SE.

**Entiéndase: Roja: Alta, Verde: Medio y Amarillo: Bajo.

\section{$\stackrel{\text { S SERVICIO RECURSO FRECUENCIA* PRIORIDAD** }}{*}$}

\begin{tabular}{|c|c|c|c|}
\hline \multirow{5}{*}{ 总 } & Agricultura & Sabana & 11 \\
\hline & Ganadería & Sabana & 47 \\
\hline & Pesca & Laguna & 51 \\
\hline & $\begin{array}{c}\text { Agua } \\
\text { (Calidad y } \\
\text { cantidad) }\end{array}$ & $\begin{array}{l}\text { Laguna y } \\
\text { Fuentes } \\
\text { hídricas }\end{array}$ & 73 \\
\hline & $\begin{array}{c}\text { Alimento } \\
\text { (Frutos) }\end{array}$ & Bosque & 42 \\
\hline \multirow{3}{*}{ 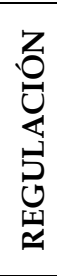 } & $\begin{array}{l}\text { Regulación } \\
\text { hídrica }\end{array}$ & $\begin{array}{l}\text { Laguna y } \\
\text { Fuentes } \\
\text { hídricas }\end{array}$ & 16 \\
\hline & $\begin{array}{c}\text { Depuración } \\
\text { de agua }\end{array}$ & $\begin{array}{c}\text { Humedal o } \\
\text { Pantano }\end{array}$ & 21 \\
\hline & $\begin{array}{l}\text { Regulación } \\
\text { climática }\end{array}$ & Bosques & 19 \\
\hline \multirow{4}{*}{ 罢 } & Recreación & Laguna & 71 \\
\hline & $\begin{array}{c}\text { Valor } \\
\text { histórico - } \\
\text { Natural }\end{array}$ & Laguna & 41 \\
\hline & $\begin{array}{c}\text { Conocimiento } \\
\text { científico }\end{array}$ & Sabanas & 73 \\
\hline & $\begin{array}{l}\text { Valor de } \\
\text { existencia }\end{array}$ & $\begin{array}{c}\text { Laguna y } \\
\text { Bosques }\end{array}$ & 73 \\
\hline
\end{tabular}

Con respecto a los ecosistemas acuáticos prestan servicios de regulación como estabilización climática y atmosférica, regulan el ciclo hídrico y la humedad meso climática, de la vida silvestre, sumideros de carbono (entre otros), de abastecimiento como agua (calidad y cantidad) y culturales (valor paisajístico y recreativo) (Vilardy \& González 2012). Sin embargo, al relacionar la prestación de servicios ecosistémicos de las fuentes hídricas, fueron considerados prioritarios los servicios de tipo culturales, debido a que la población percibe que el recurso hídrico es abundante, de buena calidad y representa una fuente inagotable con alto poder de dilución con respecto a la carga contaminante que recibe, está es una 
característica que se ha identificado como punto crucial al hablar de la gobernanza del agua (Zamudio 2009).

En cuanto a los ecosistemas terrestres en la zona se evidencio que los servicios ecosistémicos prestados por las sabanas consideradas suelos improductivos y de bajo valor (Lara \& Urrutia 2011), toman un papel relevante en la zona y se asocian en mayor medida al conocimiento científico e investigación y en menor proporción a actividades ganaderas y agricultura, las cuales esta supeditadas a los permisos dados las entidades propietarias de los predios. Así mismo, al identificar los servicios asociados a los bosques considerados como uno de los biomas más importantes del mundo en términos del área que cubren (aproximadamente 30\%), los bienes y servicios que proporcionan y la biodiversidad que contienen (aproximadamente 90\% de la biodiversidad terrestre) (Lara \& Urrutia 2011) fueron priorizados por su valor de existencia, regulación climática y protección al recurso hídrico.

\section{EVALUACIÓN DE LOS SERVICIOS}

\section{Ecológico}

Se reconocieron 9 categorías de parches, que representan diferentes estados de coberturas del ecosistema: Bosque Natural (B), Sabanas (S), Cultivos Transitorios (Ct), Cultivos Permanentes y Semipermanentes (Cps), Bosque Plantado (Bp), Laguna (L), Cuerpos de Agua (Ca), Humedal $(\mathrm{Hu})$ y Otros $(\mathrm{O})($ Tabla 2).

Tabla 2 Coberturas identificadas en la vereda Carimagua, municipio de Puerto Gaitán, departamento del Meta.

\begin{tabular}{|c|c|c|}
\hline Cobertura & Ha & $\%$ \\
\hline Bosque natural (Bn) & 3537.87 & 15.61 \\
\hline Sabana $(S)$ & 17774.64 & 78.42 \\
\hline Cultivos Transitorios (Ct) & 98.13 & 0.43 \\
\hline $\begin{array}{l}\text { Cultivos Permanentes y } \\
\text { Semipermanentes (Cps) }\end{array}$ & 90.39 & 0.40 \\
\hline Laguna (L) & 158.91 & 0.70 \\
\hline Bosque plantado (Bp) & 141.94 & 0.63 \\
\hline Cuerpos de Agua (Ca) & 90.47 & 0.40 \\
\hline Humedal (Hu) & 307.97 & 1.36 \\
\hline Otro $(\mathrm{O})$ & 466.19 & 2.06 \\
\hline TOTAL & 22666.51 & 100 \\
\hline
\end{tabular}

La evaluación con Fragstats indicó que la composición de la zona objeto de estudio está representada por sabanas y bosques de galería, mientras que la estructura del área está determinada en mayor proporción por sabanas con un
$80,80 \%$ mientras que la laguna representa la menor proporción con $0,70 \%$ indicando que los ecosistemas terrestres determinan el polígono de estudio, y actúan como fuente de investigación en la zona.

En cuanto a la función, los bosques de galería presentan una conectividad entre fragmentos equivalente a 363,69 siendo mayor a las sabanas con un 227,14 lo cual refleja que las coberturas vegetales mantienen su función de corredor biológico lo cual beneficia directamente a la biodiversidad.

Debe tenerse en cuenta que si bien los servicios ecosistémicos pueden darse en cualquier parte, no necesariamente brindan servicios de la misma calidad o cantidad en todas partes. Así, la regulación del ciclo hidrológico es un servicio de estos ecosistemas boscosos, pero tendrá valor solamente si la gente lo percibe como tal.

Adicionalmente, estos bosques contribuyen a la provisión de agua potable y a reducir el riesgo de inundaciones, si bien en la zona este servicio es importante, no contribuirá a aumentar el valor del bosque si no hay gente que se beneficie de él. De igual manera, la regulación del ciclo de carbono es un servicio ofrecido por todos los bosques, pero el servicio de mantener un clima favorable mediante el secuestro y almacenamiento de carbono es de mejor calidad en bosques con una rápida acumulación de biomasa y largos plazos de almacenamiento (Rodríguez 2002).

De esta manera, la dimensión biofísica o ecológica de los servicios se encuentra íntimamente relacionada con la biodiversidad y los ecosistemas, y su capacidad de generar servicios no depende de las preferencias humanas; sin embargo, la visualización por parte de las comunidades si permite priorizar acciones en pro de su conservación (Rodríguez 2002).

Económico. De acuerdo a los resultados de las encuestas aplicadas, para el salario en días equivalente a un jornal se determinaron los valores de: quinientos (500), mil (1 000) y mil quinientos (1 500) pesos; para el salario de medio tiempo se determinaron los valores de: tres mil quinientos pesos (3 500 ), cinco mil pesos (5 000) y seis mil quinientos pesos (6 500) y para un salario mínimo, los valores de: siete mil quinientos pesos $(7500)$, diez mil pesos (10 000) y doce mil quinientos pesos (12500).

El modelo estimó una disponibilidad a pagar promedio de \$2 206 mensuales, la mediana de la disponibilidad a pagar estimada fue de $\$ 980$ mensuales.

Con base en los resultados obtenidos se realizó la 
extrapolación al número de encuestados que fue de ciento cinco (105) personas y tomando como valor el promedio de la DAP estimada dos mil doscientos cinco pesos con noventa y cinco centavos $(\$ 2205,95)$; obteniendo un valor de DOSCIENTOS TREINTA Y UN MIL SEISCIENTOS VEINTICUATRO PESOS CON SETENTA Y CINCO CENTAVOS (\$231 624,75) mensuales; lo que representa al año la suma de DOS MILLONES SETECIENTOS SETENTA Y NUEVE MIL CUATROCIENTOS NOVENTA Y SIETE PESOS (\$2 779497$)$.

Finalmente, se realizó la extrapolación a todos los habitantes del municipio de Puerto Gaitán, tomando como base las proyección del DANE para el año 2015, que presenta una población de 18556 habitantes, que pagarían un valor mensual de CUARENTA MILLONES NOVECIENTOS TREINTA Y TRE MIL SEISCIENTOS OCHO PESOS CON DOS CENTAVOS $(\$ 40933608,2)$ y tomando como valor el promedio de la DAP estimada $(2205,95)$ para un año pagarían la suma de CUATROCIENTOS NOVEINTA Y MIL MILLONES DOSCIENTOS TRES MIL DOSCIENTOS NOVENTA Y OCHO PESOS (\$491 203 298).

En cuanto a la dimensión monetaria, está se relacionó directamente con los beneficios que los habitantes consideraron obtienen de los ecosistemas presentes en la zona de estudio, y que tienen un reflejo en el mercado o su valor monetario puede estimarse con mercados hipotéticos (pagar por disfrutar de un servicio).

Así, la disposición monetaria del valor depende no sólo de la demanda social de los beneficios generados por los servicios, sino que depende principalmente del estado de los ecosistemas y la biodiversidad (dimensión biofísica del valor) y del comportamiento ambiental, conocimiento ecológico local, e identidad local y cultural de las personas (Costanza \& Farber 2002).

En cuanto a los valores obtenidos, se infiere que la disposición a pagar es alta al ser evaluada teniendo en cuanta que: 1) Los habitantes no son propietarios de la zona y considerando que en el área se conserva y se mantiene relaciones socioecosistémicas y 2) La percepción social de que los bosques y cuerpos de agua son bienes comunes y deben ser protegidos y conservados por el estado, siendo este el responsable de su manejo limita que la sociedad reconozca la necesidad de invertir dinero personal en estas zonas.

\section{Social}

Grado de uso de los Recursos. La población asentada en el área de estudio reconoce el uso que dan al área en aspectos clasificados en cuatro grandes grupos: agua, fauna, flora y suelo (territorio y agricultura).

En cuanto a la percepción sobre los servicios y su vulnerabilidad, los servicios más utilizados del complejo ecológico asociado con la laguna Carimagua por los actores sociales implicados son por orden: (1) servicios culturales (recreación, valor histórico - natural, conocimiento científico y valor de existencia) el $97 \%$ de las personas confirmaban usar dichos servicios, (2) servicios de abastecimiento (agricultura, ganadería, pesca, agua en términos de calidad y cantidad y alimento: frutos) el $89 \%$ de las personas confirmaban usar dichos servicios) y (3) servicios de regulación (regulación hídrica, depuración de agua, regulación climática) 28\% de las personas confirmaban disfrutar de dichos servicios.

En cuanto a la vulnerabilidad, se consideró que los servicios más susceptibles a disminuir son los de abastecimiento (40\% del total de servicios percibidos como vulnerables), seguidos de los de regulación ( $34 \%$ del total de servicios percibidos como vulnerables) y los culturales ( $26 \%$ del total de servicios percibidos como vulnerables).

Al verificar los servicios identificados por los pobladores del área, se relacionan directamente con servicios que cubren necesidades básicas insatisfechas y son determinantes para poblaciones rurales (Max-Neef 1992). Por ello, la relevancia en el mantenimiento del suministro de estos servicios en calidad y cantidad ya que satisfacen las necesidades fundamentales de los actores que habitan e interactúan con el sistema, lo cual concuerda con lo reportado por Vilardy \& González para el complejo ecosistémicos de la ciénaga grande de Santa Marta (Vilardy \& González 2011).

Los actores sociales consideran que específicamente, son los servicios culturales (valor histórico natural, investigación y recreación) son los más utilizados y con tendencia a aumentar su uso, mientras que los servicios de abastecimiento son los más vulnerables debido a la incorporación de nueva población y sistemas productivos (Tabla 3).

Al evaluar los servicios ecosistémicos percibidos por los actores del área, se evidencia que la tendencia es la disminución de los servicios asociados a los ecosistemas acuáticos, esto se debe principalmente por la expectativa de incorporación de sistemas productivos y nuevos asentamientos.

Adicionalmente, se evidencia que los servicios de pesca, calidad y cantidad de agua y conocimiento científico son considerados de importancia internacional por estar asociados a la macrocuenca del Orinoco. 
El método de integración de servicios ecosistémicos de aprovisionamiento y culturales basada en percepciones sociales permite visualizar en el territorio áreas que hacen parte de la cotidianidad y determinan sus interacciones con el medio que se traduce en el bienestar de los habitantes locales (MEA 2005). Por ello, la gestión del territorio está focalizada en uno o pocos servicios de abastecimiento, lo que supone una reducción en los servicios de regulación y en la biodiversidad que los suministran. Esto es debido a que, en la mayoría de los casos, los servicios de regulación y culturales que no tienen un reflejo en el mercado no son considerados en la toma de decisiones (Martín-López et al. 2010).

Tabla 3. Tendencia de los servicios de los ecosistemas suministrados por el complejo ecológico asociado a la laguna Carimagua, escala de los beneficiarios de dichos servicios. $(\uparrow$ : aumenta; $\leftrightarrow$ : se mantiene; $\downarrow$ : disminuye. ${ }^{* *}$ Escala a la que se demanda y usa el servicio.

\begin{tabular}{|c|c|c|c|c|c|c|}
\hline \multirow[t]{2}{*}{ Tipo } & \multirow{2}{*}{$\begin{array}{c}\text { Servicio } \\
\text { del } \\
\text { Ecosistema }\end{array}$} & \multirow[t]{2}{*}{ Tendencia } & \multicolumn{4}{|c|}{ Beneficiarios de los Servicios $^{* *}$} \\
\hline & & & Local & Regional & Nacional & Internacional \\
\hline \multirow{7}{*}{ 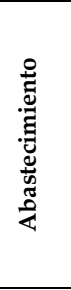 } & Agricultura & $\widehat{0}$ & & & & \\
\hline & Ganadería & $\uparrow$ & & & & \\
\hline & Pesca & $\downarrow$ & & & & \\
\hline & $\begin{array}{l}\text { Calidad de } \\
\text { agua }\end{array}$ & $\sqrt{ }$ & & & & \\
\hline & Cantidad & ? & & & & \\
\hline & de agua & $\sqrt{t}$ & & & & \\
\hline & $\begin{array}{l}\text { Alimento } \\
\text { (Frutos) }\end{array}$ & $\sqrt{ }$ & & & & \\
\hline \multirow{4}{*}{ 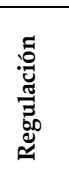 } & Regulación & $\Leftrightarrow$ & & & & \\
\hline & $\begin{array}{l}\text { Hídrica } \\
\text { Depuración }\end{array}$ & & & & & \\
\hline & de agua & $\downarrow$ & & & & \\
\hline & $\begin{array}{l}\text { Regulación } \\
\text { Climática }\end{array}$ & 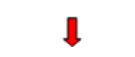 & & & & \\
\hline \multirow{7}{*}{$\begin{array}{l}\frac{0}{\frac{0}{\pi}} \\
\frac{\overparen{J}}{J} \\
\frac{0}{J}\end{array}$} & Recreación & Ð & & & & \\
\hline & Valor & & & & & \\
\hline & Histórico & $\widehat{\imath}$ & & & & \\
\hline & Natural & & & & & \\
\hline & $\begin{array}{l}\text { Conocimiento } \\
\text { Científico }\end{array}$ & $\widehat{\imath}$ & & & & \\
\hline & Valor de & & & & & \\
\hline & Existencia & & & & & \\
\hline
\end{tabular}

Sin embargo, en cuanto a las relaciones de gobernanza y manejo integral del territorio, es necesario ampliar el enfoque metodológico al total de actores del área, con el fin de identificar servicios ecosistémicos presentes y aprovechados por las comunidades de manera directa e indirecta como lo son los relacionados con servicios de regulación, ya que se evidencio que el ecosistema presta servicios asociados a la regulación climática y depuración de agua que no son percibidos como importantes por los habitantes debido a que no se usan de manera directa, lo cual concuerda con lo reportado por Vilardy \& González (2011) para ecosistemas lenticos neotropicales.
En cuanto a la selección de los servicios "claves" se identificó que existe gran expectativa tanto institucional como por la comunidad, por la conservación de los mismos, considerando que puede llegar a constituir una gran fuente de alimento y el potencial en producción de conocimiento para la Altillanura Orinoquence.

\section{GESTIÓN Y PLANIFICACIÓN DEL TERRITORIO}

\section{Juego de Roles}

Los resultados obtenidos reflejan que al tener la opción de ser propietarios de $27 \mathrm{Ha}$ y con la necesidad de incorporar sistemas productivos en por lo menos $70 \%$ de predio (según las normas establecidas), se evidenció que el 100\% de los jugadores a un escenario de diez años, amplió la incorporación de actividades productivas más allá del mínimo establecido.

Al aplicar el juego a medida que los jugadores recibían divisas por el cambio de uso del suelo, fueron aumentando paulatinamente la cantidad de dividendos, sin tener en cuenta los servicios ecosistémicos que perdían, agotaban o alteraban.

En el juego se evidenció que los habitantes actuales del área de influencia directa de estudio conservan la tendencia a incorporar sistemas ganaderos en sus suelos, esto asociado a la tradición de la zona, riqueza de agua y presencia de mercados donde comercializar el ganado.

Las actividades asociadas a la protección de bosques se reportaron decrecientes al paso de los años, pasaron de $28 \mathrm{Ha}$ equivalentes a $25,92 \%$ de territorio en el primer año a $12 \mathrm{Ha}$ correspondientes a $11,11 \%$, lo cual se debe a que el bosque no les proporciona ingresos económicos y no está asociado a mercados.

En cuanto al comportamiento como grupo, el agua no fue considerada un factor determinante, lo cual se asoció directamente a la riqueza hídrica de la zona, aunque no se colocó límite a este recurso si se estableció que era necesaria su presencia para la viabilidad del territorio.

Se evidenció que el año que determinó el cambio del uso del suelo, está asociado al quinto año debido a las ganancias obtenidas, punto en el cual los participantes olvidaron que el condicionamiento que indicaba que solo era necesario utilizar el 70\% del territorio para garantizar la tendencia de la tierra. A su vez, se evidenciaron tensiones cotidianas entre los 
propietarios ya que al ver que su vecino recibía mayores ingresos por incluir determinada actividad, los vecinos la aumentaban en la siguiente ronda para su predio.

Al evaluar los resultados obtenidos con los participantes se logró determinar el proceso de relacionamiento social y los vínculos entre la incorporación de actividades, la conservación y el ingreso económico que pueden llegar a obtener los beneficiarios de un proceso de titulación.

El resultado más representativo fue el análisis de los participantes al ver que el comportamiento individual los llevaría a la perdida de los bosques y disminución de la calidad y cantidad del recurso hídrico, para lo cual acordaron que era necesario blindar el área para garantizar el manejo colectivo del territorio y prestación de servicios ecosistémicos que en la actualidad les brindan bienestar.

\section{Selección de la categoría según el decreto 2372 de 2010}

La selección se enmarca en el análisis de los elementos biofísicos, ecológicos, socioeconómicos y culturales analizados, en los cuales se identifican y evalúan los Servicios Ecosistémicos prestados por la zona, resaltando su condición natural y su representatividad, grado de amenaza, irremplazabilidad e integridad ecológica, así como la importancia social y cultural dentro del territorio.

Entre los servicios ecosistémicos que presta la zona sobresalen los asociados a procesos hidrológicos, como la recarga de acuíferos, el agua acumulada desciende hasta las napas subterráneas, retención, transformación y/o remoción de sedimentos, nutrientes y contaminantes los cuales permiten regular el clima, activar los ciclos de la materia y garantizar la calidad y cantidad del recurso hídrico. Otro factor relevante es que el ecosistema presenta una alta productividad, albergando numerosas poblaciones que permiten garantizar la sobrevivencia de especies, la polinización, fuente de alimento y un gran valor cultural por su historia.

De acuerdo con las características del territorio, se determinó la categoría que se asimilan a las condiciones del área propuestas en el Decreto 2372 de 2010 y se identificó la viabilidad para el complejo ecológico asociado con la laguna Carimagua, bajo la categoría de Distrito de Manejo Integrado el cual consiste en un espacio geográfico, en el que los paisajes y ecosistemas mantienen su composición y función, aunque su estructura haya sido modificada y cuyos valores naturales y culturales asociados se ponen al alcance de la población humana, para destinarlos a su uso sostenible, preservación, restauración, conocimiento y disfrute .
La sostenibilidad de la categoría de protección en el área, se encuentra asociada directamente a los recursos recaudados por los habitantes, los cuales permitirán generar conocimiento e incorporar un sistema de administración social participativa.

\section{CONCLUSIONES}

- La identificación de servicios ecosistémicos por parte de los actores locales se fundamenta en su relación con los ecosistemas y el paisaje, su cotidianidad y su reconocimiento como parte del Territorio, más que en un recurso natural determinado, en este caso el agua.

- El grado de identificación y conocimiento de los actores locales sobre su entorno constituye la base de la percepción clara sobre servicios ecosistémicos ofertados y la necesidad de mantenerlos, especialmente los culturales asociados al valor de existencia.

- La metodología utilizada permitió la evaluación integral de los servicios ecosistémicos, evidenciando el conocimiento y gobernanza que tienen los actores sociales sobre el manejo del territorio y la importancia de generar procesos participativos en el área.

- La Evaluación integral de los Servicios ecosistémicos implica reconocer la complejidad del sistema y las múltiples interacciones que emergen entre los actores sociales y su entorno, lo cual facilita el diseño de estrategias para la planificación ambiental territorial.

\section{REFERENCIAS}

BALVANERA, P. \& H. COTLER (eds). 2009. Estado y tendencias de los servicios ecosistémicos. En: Capital natural de México, vol. II: 185-245. Estado de conservación y tendencias de cambio. Conabio, México.

CÁRDENAS, J. C. CASTAÑEDA, J. L. CASTILLO, D. LAVERDE, C. PEREIRA, M.F. \& L.A. RODRÍGUEZ. 2013. Métodos complementarios para la valoración de la biodiversidad: una aproximación interdisciplinar. Instituto de Investigaciones de Recursos Biológicos Alexander von Humboldt y Universidad de los Andes. 168 pp.

CENTRO DE INVESTIGACIONES EN ECOSISTEMAS. CEECO. 2012. México.

COSTANZA, R. \& S. FARBER. 2002. Introduction to the 
special issue on the dynamics and value of ecosystem services: integrating economic and ecological perspectives. Ecological Economics 41(3): 367-373.

DE GROOT, R. WILSON, M.A. \& R.M.J. BOUMANS. 2002. A typology for the classification, description and valuation of ecosystem functions, goods and services. Ecological Economics 41(3): 393-408.

GÁMEZ, M. LÓPEZ, I. \& S. MOLNÁR. 2008. Monitoring environmental change in an ecosystem. BioSystems 93: 211217.

LARA, A. \& R. URRUTIA. 2011. Servicios ecosistémicos de los bosques nativos en Chile: estado del arte y desafíos. En: Valoración de servicios ecosistémicos: Conceptos, herramientas y aplicaciones para el ordenamiento territorial. $741 \mathrm{pp}$.

LAYKEA, C. MAPENDEMBE, A. BROWN, C. WALPOLE, M. \& J. WINN. 2012. Indicators from the global and subglobal Millennium Ecosystem Assessments: An analysis and next steps. Ecological Indicators 17: 77-87.

MARTÍN-LÓPEZ, B., GARCÍA-LLORENTE, M. GÓMEZBAGETHUM, E. MONTES, C. 2010. Evaluación de los servicios de los ecosistemas del sistema socio-ecológico de Doñana. Forum de Sostenibilidad. Revista de la Cátedra UNESCO sobre Desarrollo Sostenible de la UPV/EHU. 91111.

MAX-NEEF M.A. 1992. Development and human needs. En: EKINS P. \& MAX-NEEF M.A. (eds). Real-life Economics: Understanding Wealth Creation. Routledge. 197-214.

MCGARIGAL, K. CUSHMAN, SA. \& E. ENE. 2012. FRAGSTATS v4: Computer software program produced by the authors at the University of Massachusetts, Amherst. URL Available at the following web site web: http://www.umass.edu/landeco/research/fragstats/fragsta ts.html

MEA. 2005. Ecosystems and human well-being: current state and trends. Island Press, Washington, DC.

PRAGUER, K. REED, M. \& A. SCOTT. 2011. Encouraging collaboration for the provision of ecosystem services at a landscape scale-Rethinking agri-environmental payments. Land Use Policy.

RODRÍGUEZ, J. 2002. Los servicios ambientales del bosque: el ejemplo de Costa Rica. Revista Forestal Centroamericana. 37:
47-53.

VILA, J. VARGA, D. LLAUSÀS, A. \& A. RIBAS. 2006. Conceptos y métodos fundamentales en ecología del paisaje (landscape ecology). Una interpretación desde la geografía. Doc. Anàl. Geogr. 48: 151-166.

VILARDY, S. \& J.A. GONZÁLEZ (eds.). 2011. Repensando la Ciénaga: Nuevas miradas y estrategias para la sostenibilidad en la Ciénaga Grande de Santa Marta. Universidad del Magdalena y Universidad Autónoma de Madrid. Santa Marta, Colombia. 228 pp.

ZAMUDIO, R. 2009. Gobernabilidad sobre el recurso hídrico en Colombia: entre avances y retos. Revista Gestión y Ambiente 15(3): 99-112. 